\title{
Nuevos horizontes para las finanzas internacionales. Las propuestas del Pontificio Consejo Justicia y Paz
}

\section{Paolo Foglizzo'}

\begin{abstract}
En nuestra entrega anterior ( $n^{\circ} 264$, octubre-diciembre de 2011 ) publicamos como documento la Nota del PONTIFICIO CONSEJO DE JUSTICIA Y PAZ Para una reforma del sistema financiero y monetario internacional en la perspectiva de una autoridad pública con competencia universal seguida de dos comentarios de lldefonso Camacho Laraña y de Jaime Loring Miró. En este número aparece, continuando el debate abierto por aquel documento, una colaboración del economista italiano Paolo Foglizzo, publicada en la revista social de los jesuitas editada en Milán, Aggiornamenti Sociali con la que nos une una fraterna cercanía desde hace tiempo. Creemos contribuir de este modo a la difusión y discusión de un documento del pensamiento social cristiano con gran incidencia y relevancia actual.
\end{abstract}

Un reciente documento del Pontificio Consejo Justicia y Paz propone una reforma del sistema financiero global dirigida a institucionalizar una autoridad pública con competencia universal. ¿En qué se basa dicha respuesta?, ¿̇qué pasos tendrían que darse para ponerla en práctica?, ¿qué elementos de nuestra realidad quedan manifiestos si la observamos desde esta perspectiva?

' Redacción de Aggiornamenti Sociali. La versión original italiana, en Aggiornamenti Sociali 63 (2012)

117-125. foglizzo.p@aggiornamentisociali.it. La traducción de la misma ha sido realizada por Adriana García Lupato, servicio de investigación, ETEA-Loyola, Sevilla. 
El 24 de octubre de 2011, el Pontificio Consejo Justicia y Paz presentó la Nota Para una reforma del sistema financiero y monetario internacional desde la perspectiva de una autoridad pública con competencia universal: una propuesta para enfrentarse a las causas que desencadenaron la crisis financiera global, con la idea además de contribuir a la cumbre del G20 que se desarrolló poco tiempo después (Cannes, Francia, 3 y 4 de noviembre de 2011).

A través de esta Nota, el dicasterio vaticano desarrolla su propia misión de difundir, profundizar y contribuir a la experimentación de la doctrina social de la Iglesia (Toso 2012, $\mathrm{n}^{\circ} 1$ ), iluminando a través de la misma los problemas surgidos a nivel global y reflejando su actualidad. Es lo que ya sucedió en el pasado con los temas de la deuda externa de los países empobrecidos, del racismo, del comercio internacional de armas, de la reforma agraria y de la corrupción. Cabe señalar que ésta no es la primera vez que el Pontificio Consejo Justicia y Paz centra su atención en cuestiones económicas y financieras, como lo demuestran una serie de publicaciones y seminarios internacionales (PONTIFICIO CONSEJO JUSTICIA y PAZ 1994a; 1994b; de SALINS Y DE VILLEROY DE GALHAU 1994) y sobre todo, la nota más reciente publicada con vistas a la Conferencia de Doha del 2008 (PONTIFICIO CONSEJO JusticIA Y PAZ 2008).

El documento del pasado octubre se engloba por lo tanto en una amplia vía de investigación, reflexión y propuestas, pero sobre todo se basa en el magisterio pontificio, en particular en las encíclicas Pacem in terris (1963) y Caritas in veritate (2009), de las que especifica y concreta el mensaje en el contexto de la actual situación del sector financiero internacional. En estas páginas presentamos brevemente el contenido, para señalar posteriormente qué contribución puede ayudar a la comprensión de algunas situaciones concretas y concluir con el examen de algunas de las reacciones que dicho documento ha suscitado.

${ }^{2}$ Disponible en www.justpax.va; traducción al castellano en RFS 66 (2011) 753-786. 


\section{Documentos del Pontificio Consejo Justicia y Paz}

Los textos que en el transcurso de los años el Pontificio Consejo de Justicia y Paz, denominado Comisión Pontificia lustitia et Pax hasta junio de 1988, ha dedicado a temas de actualidad de ámbito global, tomando como punto de partida la doctrina social de la lglesia son:

- Comisión Pontificia lustitia et Pax (1986) Al servicio de la comunidad humana: una consideración ética de la deuda internacional.

- Comisión Pontificia lustitia et Pax (1988) La Iglesia ante el racismo. Para una sociedad más fraterna.

- PONTIFICIO CONSEJO JUSTICIA Y PAZ (1994) El comercio internacional de armas. Una reflexión ética.

- Pontificio consejo justicia y paz (1997) Para una mejor distribución de la tierra. El reto de la reforma agraria.

- Pontificio consejo justicia y paz (2006) La lucha contra la corrupción.

\section{El contenido del documento}

La primera preocupación del documento es definir su propia perspectiva: se ponen de relieve dos palabras claves como son "responsabilidad" y "bien común". Se trata de un pasaje fundamental, en cuanto expresa la intención en la que se basan el análisis y la argumentación: es de verdad distinto razonar sobre el sistema financiero internacional en esta óptica, o en aquélla correspondiente a la maximización del beneficio de algunos operadores o países.

\section{I.I. Las causas de la crisis}

El primer párrafo de la Nota se dedica a una breve y lúcida reconstrucción de la crisis que estamos atravesando, cuya causas se encuentran en una combinación de errores técnicos y de responsabilidades morales $\left(n^{\circ} 1\right)$; de la que proviene la espiral de expansión del crédito y de la moneda ${ }^{3}$, que ha tenido como consecuencia una

${ }^{3}$ Para un breve análisis, pero técnicamente muy conseguido, de dicha dinámica, se aconseja leer PALAISROYAL INTIATIVE 2011 . Se trata de un documento de gran prestigio: la iniciativa Palais-Royal es un grupo 
serie de burbujas especulativas y sobre todo, de un incremento desproporcionado de los riesgos asumidos por las instituciones financieras internacionales más importantes. Cuando la situación precipitó, a partir de la quiebra de Lehmann Brothers en septiembre de 2008 (Foguzzo 2008), la caída en la confianza ha llevado a los gobiernos a tomar enormes medidas para salvar al sistema bancario aunque esto no haya evitado el contagio de la crisis a la economía real, con graves consecuencias para el empleo.

Todo esto se ha producido por un liberalismo económico sin reglas ni supervisión $\left(n^{\circ} 1\right)$, una verdadera ideología que exagera algunos aspectos de las leyes de funcionamiento de los mercados, favoreciendo a aquellos países y operadores que se encuentran en una posición de ventaja en el tablero económico global. De ahí que se produzca el reclamo, ya indicado en Caritas in veritate (n. 45), a una economía que ya no se base en el utilitarismo individualista, sino en una ética amiga de la persona y en un espíritu de solidaridad.

\subsection{La finanza como técnica}

La importancia de la recuperación de una base ética para el sistema económico y financiero aumenta según se constata cómo la aplicación de los progresos informáticos ha logrado que las finanzas tengan una técnica particularmente sofisticada. Aumenta por lo tanto el riesgo de caer en una "falacia tecnicista", es decir, considerar que los problemas a los que nos enfrentamos son de carácter exclusivamente técnico (n. 2), minimizando el valor de las elecciones de los operadores, que son y siguen siendo personas humanas.

La negativa a un "algo más", entendido como un valor añadido a la técnica, no sólo imposibilita encontrar soluciones adecuadas para los problemas, sino que empobrece cada vez más, tanto en el plano material como en el moral, a las principales víctimas de la crisis (n. 2).

internacional de ex ministros, gobernadores de bancos centrales y otros funcionarios de instituciones nacionales e internacionales, reunido bajo invitación de Michael Camdessus (Gobernador del Banco de Francia de 1984 a 1987, Director del Fondo Monetario Internacional de 1987 a 2000), Alexandre Lamfalussy (Director del Banco de Regulación Internacional de 1985 a 1993 y Presidente del Instituto Monetario Europeo, antesala del Banco Central Europeo, desde su fundación en 1994 a 1997) y Tommaso Padoa-Schioppa (Ministro de Economía de Italia de 2006 a 2008 y durante mucho tiempo funcionario de altísimo nivel del Banco de Italia, del Banco Central Europeo y del Fondo Monetario Internacional (cfr. BOORMAN e ICARD 2012). 
Solamente ese "algo más" permite recuperar la prioridad de la ética sobre la economía, y el sentido de pertenencia común a la familia humana en el que se cimenta la solidaridad, también en el desarrollo de la actividad financiera.

Se trata de una estimulante aplicación a la economía y a las finanzas de uno de los puntos cardinales del magisterio de Benedicto XVI, que ha insistido varias veces sobre los riesgos de la deriva tecnicista de nuestra cultura: la adoración de la técnica, a la que se deben las mejoras de las condiciones de vida registradas en los últimos dos siglos, lleva a pensar que sea lícito todo lo que es técnicamente posible. Esta mentalidad conlleva riesgos y consecuencias funestas en todos los ámbitos de la vida, no sólo en el campo de la bioética, donde esto se manifiestan de forma muy patente.

No es siquiera un destello que se pueda señalar únicamente a partir del pensamiento de la Iglesia. En 2002 -mucho antes del comienzo de la crisis- Warren Buffet, el gran financiero estadounidense, definía los derivados como armas financieras de destrucción masiva ${ }^{4}$. El corolario a esta posición debería ser que su utilización tendría que tener las mismas medidas de control, éticas y políticas, y los mismos controles en su empleo, que los arsenales nucleares. El desarrollo de la actual crisis nos demuestra que no se ha llevado a cabo de esta forma.

\section{I.3. La propuesta de una autoridad pública mundial}

En este marco se pone de relieve cuál es el fundamento de la propuesta de creación de una autoridad pública mundial que se indica en la Nota. En la doctrina social de la Iglesia esto no es una novedad: ya en 1963 Juan XXIII le había, proféticamente, dedicado el capítulo IV de la Pacem in terris, retomada por Benedicto XVI en Caritas in veritate (n. 67).

El razonamiento es relativamente sencillo: la creciente interdependencia entre los países manifiesta el nacimiento de una auténtica comunidad política mundial, que, en cuanto tal, tiene su propio bien común, que consiste en que todas aquellas cuestiones, cada vez más presentes en la agenda internacional, que no pueden ser tenidas en cuenta de forma eficaz a nivel nacional: paz, desarme, migraciones, ambiente, seguridad alimentaria, tutela de los derechos humanos, economía y políticas de desarrollo, etc. La búsqueda de este bien común requiere, según la

${ }^{4}$ W. Buffetr, Carta a los accionistas de Berkshire Hathaway Inc., 2002 www.berkshirehataway.com/ letters/2002.pdf, 15. "Financial weapons of mass destruction", en el original inglés. 
tradición católica, de la creación de una autoridad dotada con los poderes necesarios para hacerse cargo de la misma.

Para conseguir dicho resultado no son estructuralmente adecuadas todas las formas de coordinación y acuerdo internacionales, comenzando por la diplomacia y el multilateralismo, aunque sean de fundamental importancia: ninguno de los actores que en ellas participan son auténticamente "super partes", y por lo tanto, más que el bien común universal lo que tienen como objeto es la tutela de intereses particulares (nacionales o de grupos de países). Las organizaciones internacionales que siguen el modelo de Naciones Unidas, aunque hayan logrado acercamientos importantes, no tienen la autoridad requerida sobre los propios miembros, mientras que a las formas de coordinación como el G7 o el G205, les falta representatividad necesaria, ya que excluyen a muchos países y corren el riesgo de funcionar como un club que tutela los intereses de los propios miembros (n. 4).

Por tal motivo, la Nota precisa que es necesario el paso de las actuales formas de gobernanza internacional (coordinación horizontal sin una autoridad super partes) a un verdadero y propio "gobierno compartido"6: un sistema que, además de la coordinación horizontal, establezca una autoridad "super partes", funcional y proporcional al gradual desarrollo de una sociedad política mundial (n. 3). Notamos además un signo de la preferencia tradicional que la doctrina social tiene hacia la posibilidad de identificar con claridad aquéllos a los que corresponde la promoción del bien común, y que por lo tanto son responsables ${ }^{7}$, frente a las formas impersonales o automáticas -entre ellas pensamos inmediatamente en la "mano invisible"- que según otros posicionamientos son capaces de alcanzar el mismo resultado.

Para evitar problemas de interpretación tanto imperialista como "orwelliana", la Nota, siguiendo la estela de Pacem in terris, es extremadamente concreta en el dibujo de la fisionomía de esta autoridad pública mundial. En primer lugar, debe ser fruto de un acuerdo libre y compartido, que excluya cualquier forma de coacción: su creación requerirá por lo tanto de tiempo y realizarla de forma gradual. Es fundamental la construcción de un clima de confianza, basado en el respeto

\footnotetext{
${ }^{5}$ El paso del G7 al G20 se valora de todos modos como una evolución positiva (n 4). Sobre el funcionamiento del G20, cfr. FoGuzzo 2010.

${ }^{6}$ Shared governement, en inglés en el original (ndt)

7 En el posicionamiento en que se basa Pacem in terris, queda claro que por autoridad pública mundial se entiende las instituciones $y$, dentro de las mismas, las personas, oportunamente elegidas, a las que se les otorga el deber de la promoción del bien común universal.
} 
de la diversidad entre países, que pueda garantizar la legitimidad de la nueva institución. Además, la autoridad política mundial tendrá que operar en el respeto del principio de subsidiariedad (es decir, actuando en la eliminación de aquellos obstáculos que impidan el pleno desarrollo de la potencialidad de todos los países, de todos los pueblos y de todas las personas, sin interferir en las respectivas esferas de autonomía) y visibilizando la solidaridad que les une.

\subsection{La reforma del sistema financiero global}

La ausencia de un auténtico gobierno ${ }^{8}$ se manifiesta también en la economía y las finanzas internacionales, a causa del gradual debilitamiento de la eficiencia de las instituciones financieras internacionales nacidas con los acuerdos de Bretton Woods de 1944 (n. 4) . Un segundo elemento es la ausencia de un corpus mínimo compartido de reglas necesarias para la gestión del mercado financiero global (n. 4).

Con esta situación es necesario dar inicio a un proceso de profunda reflexión y de reformas, recorriendo vías creativas y realistas, que tiendan a valorar los aspectos positivos de las instituciones y de los foros ya existentes (n. 4). Por lo tanto, no se empieza desde cero, sino que podemos construir todo lo que sea indispensable a partir de las experiencias positivas ya realizadas. La Nota señala dos: la primera es la lógica de paz, coordinación y prosperidad común, que son las bases de los acuerdos de Bretton Woods, y en particular del Fondo Monetario Internacional (FMI), cuyos Estatutos tienen un potencial probablemente todavía inexplorado. La segunda, de ámbito regional, es el Banco Central Europeo. No es casualidad que, junto a la Unión Europea y al "Fondo de Rescate", sean las instituciones a las que nos dirigimos para dar soluciones a la crisis de deuda soberana de los países de la zona Euro, a partir de Grecia. La idea es poder llegar a una institución que sea una especie de "Banco Central Mundial", encargada de la regulación de los flujos monetarios globales siguiendo la estela de los bancos centrales nacionales ${ }^{10}$.

\footnotetext{
${ }^{8}$ Se considera necesario precisar que, según la Nota, por el término "gobierno" no se quiere definir un órgano en el que se concentre un poder mundial, sino el resultado de una actividad de instituciones que puedan garantizar, a nivel mundial, la acción equilibrada que en los sistemas democráticos se desarrolla mediante la articulación de la función legislativa, ejecutiva y judicial, asegurando en su conjunto en el gobierno eficaz de la comunidad política.

${ }^{9}$ Cfr. Palais-Royal Initiative 2011 , n. 4, aunque éste se manifieste en términos de gobernanza.

10 PaLAIS-RoYAl INITIATIVE 2011, pp. 17-19, señala incluso la oportunidad de llegar a una moneda no nacional, desarrollando el instrumento representado por los derechos especiales de giro del FMI.
} 
En el camino hacia la reforma del sistema monetario y financiero internacional tiene también cabida la formulación de algunas propuestas concretas de rápida aplicación: medidas de tasación de las transacciones financiarías (crf. BECCHETTI y CIAMPOL 2011); formas de recapitalización de los bancos condicionadas al uso y apoyo de la economía real y no de la especulación financiaría; una distinción más clara entre el ámbito de la actividad de crédito convencional y la de los bancos de inversión ${ }^{11}$. Son los asuntos actuales, especialmente en las cumbres europeas.

\section{Temas actuales}

Hablar de autoridad pública mundial puede parecer incluso algo etéreo en relación con los graves problemas por los que estamos pasando. No es así: el argumento desarrollado por la Nota contiene elementos que nos permiten leer en una clave distinta las dinámicas en curso.

Un primer ejemplo se refiere directamente a ltalia. En los últimos meses de 2011 muchos se han referido a una "intervención" de Italia, objeto de un dictado por parte de otros países europeos y de varias visitas de inspección por parte de la UE y del FMI (COSTA 2012). Un párrafo de la Nota explica esta situación bajo una perspectiva distinta:

Según la lógica de la subsidiaridad, la autoridad superior ofrece su subsidium, es decir, su ayuda, cuando la persona y los actores sociales y financieros son intrínsecamente inadecuados o no logran realizar por sí mismos lo que les es requerido (n.3).

Es innegable que, con respecto a la gestión del propio déficit y deuda pública, Italia no podía realizar lo requerido, aunque esto se deba -así se espera- a causa de factores contingentes y no de una inadecuación intrínseca. En esta situación, la intervención de una autoridad superior, aunque dura en el trato, ha representado una ayuda de la que Italia se beneficia, de cara a evitar un empeoramiento mayor que podría llevar a resultados potencialmente peligrosos para Italia y no sólo para ella.

El segundo ejemplo se refiere a los países pobres. En la Nota, así como en todos los documentos del Pontificio Consejo Justicia y Paz, es evidente que la atención principal se dirige a su situación: a ellos les afecta de forma mucho más grave los costes de la crisis (n. 1) y son estos países los que se excluyen de las formas de

${ }^{11}$ Investment banking, en inglés en el original (ndt). 
coordinación del tipo G7, no G20 (n. 4). Para su tutela se afirma que las decisiones de la autoridad mundial ideada no deberán ser el resultado del pre-poder de los países más desarrollados sobre los países más débiles (n. 3).

Entre los problemas más graves a los que estos países se tienen que enfrentar hoy en día se encuentra la volatilidad de los precios de los productos alimentarios de base (en particular los cereales), que deriva también de la aplicación al mercado agrícola de las técnicas especulativas típicas de los mercados financieros, como se denuncia por parte de algunas campañas de la sociedad civil (www.sullafamenonsispecula.org). Los aumentos de precio desproporcionados registrados a partir de 2007, seguidos posteriormente por bruscas disminuciones, ponen en situación de riesgo la seguridad alimentaria de millones de personas, de tal forma que la FAO (Organización de Naciones Unidas para la alimentación y la agricultura) ha lanzado un programa de "monitoreo" de la evolución de los mercados (ref. www.fao.org/isfp) y el G20 sobre la agricultura (París, 22-23 de junio de 2011) se ha dedicado a este asunto. Aunque la Nota no lo menciona explícitamente, la cuestión se encuentra en el primer puesto de la lista de todas aquéllas de las que una autoridad mundial se tendría que ocupar y pone de manifiesto la urgencia de su creación.

\section{Para profundizar la reflexión}

La publicación de la Nota ha suscitado, de forma también inesperada, notables reacciones. Algunas voces, particularmente provenientes de ambientes conservadores del catolicismo estadounidense, evidentemente insatisfechas por las dudas expresadas sobre las virtudes del mercado dejado a sí mismo, han tratado de disminuir la importancia y la autorización del documento ${ }^{12}$, insinuando incluso que entrarían en contradicción con Caritas in veritate. Tanto, que el Secretario del Pontificio Consejo, Mario Toso, ha tenido que intervenir para matizar ulteriormente la cuestión (2012). No es una novedad: ya anteriormente los mismos ambientes habían manifestado una sorpresa similar precisamente sobre la recién publicada Caritas in veritate ${ }^{13}$.

\footnotetext{
${ }^{12}$ En Italia estas posiciones han tenido su eco en algunas intervenciones que han aparecido en el blog del vaticanista SANDRO MAGISTER: http://chiesa.espresso.repubblica.it.

${ }^{13}$ Un ejemplo de esta extraña "hermenéutica" lo representa WEIGLE G., Caritas in Veritate in Gold and Red, 7 de julio de 2009, en www.nationalreview.com. El texto de la encíclica está diseccionado en partes "auténticas", que se tienen que conservar, y partes "apócrifas" que se tienen que expurgar. El
} 
Evidentemente, la Nota toca un punto sensible entre aquéllos que apoyan el dogmatismo del libre mercado. La cuestión se dirige directamente hacia el significado de libertad del que éste se enorgullece. Si según la óptica neoliberal ésta es la ausencia de vínculos, para la teología cristiana la libertad es principalmente la capacidad de hacer el bien, mientras que llevar a cabo el mal es esclavitud. En este sentido, la falta de reglas y controles eficaces que consiente a una institución financiera la asunción de riesgos que van más allá del límite de la prudencia para transmitirlos después a otros sujetos trámite, por ejemplo, operaciones de titulización, no puede ser considerada una forma de libertad. Como concluye el analista financiero inglés Angus SIBLEY (2011),

la Iglesia Católica, en los recientes decenios, no ha promocionado su propia y segura doctrina sobre temas económicos con suficiente vigor. Si lo hubiese hecho, los economistas ultraliberales habrían encontrado unas mayores dificultades en la difusión de sus teorías profundamente erróneas, cuyas trágicas consecuencias hoy caen sobre nuestras cabezas como una maldición: pésima gestión en el campo financiero, aumento del desempleo y de la desigualdad, amenazas al ambiente ${ }^{14}$.

Consideramos pertinente una segunda reflexión. Contrariamente a una idea bastante difundida, no existen mercados sin reglas, a partir de la disciplina de los contratos que se encuentran en la base de las transacciones. Si su determinación se deja al puro "consenso de las partes", las reglas darán preferencia inevitablemente a los intereses de aquellas partes que hayan participado en su elaboración, y de entre todas ellas, a las más poderosas. Sólo una autoridad verdaderamente super partes podrá tener de verdad en cuenta el punto de vista de todos aquellos sobre los que la aplicación de las reglas tendrá un impacto: en nuestro caso, la mayor parte de la población mundial que no tiene acceso a los mercados financieros, pero que está sometida a las consecuencias de sus oscilaciones. Incluso en el área económica sólo un gobierno eficaz es capaz de producir una democracia efectiva.

resultado evidentemente satisface las preferencias del comentarista, pero con el problema que no llega a explicar cómo el autor del texto -iy se trata de Benedicto XVI!- habría aceptado publicar bajo su propio nombre partes "no auténticas".

${ }^{14}$ Loc. cit. 
Magisterio:

- Benedicto XVI (2009) Caritas in Veritate.

- JuAN XXIII (1963) Pacem in terris.

- Pontificio Consejo Justicia y Paz (2004) Compendio de la doctrina social de la lglesia, Roma 2005 Libreria Vaticana (o Madrid-Barcelona 2005, Católica (BAC) - Planeta).

- Pontificio Consejo Justicia y Paz (2008) Un nuevo pacto financiero internacional. Nota sobre la financiación del desarrollo en vísperas de la Conferencia promovida por la Asamblea General de las Naciones Unidas en Doha.

- Pontificio Consejo Justicia y Paz (2011) Por una reforma del sistema financiero y monetario internacional en la perspectiva de una autoridad pública con competencia universal.

Textos de Referencia:

- BeCCHetti, L. y CiAmpol, N. (2011) "«Zerozerocinque». Una tassa sulle transazioni finanziarie": Aggiornamenti Sociali 62, 502-511.

- Boorman, J.T. e ICARD, A. (2012) Reform on the International Monetary System. The Palais Royal Initiative, Thousand Oaks (California, EEUU), Sage.

- Costa, G. (2012) "Una nuova Europa per l'euro": Aggiornamienti Sociali 63, 7-12.

- Czerny, M. (2011) "Crisi e governance internazionale. Verso un mondo inteso come comunità di comunità": Aggiornamenti Sociali 62, 99-106.

- Foglizzo, P. (2004) "Finanza internazionale e agire morale. Un sussidio pastorale": Aggiornamenti Sociali 55, 292-299.

- Foglizzo, P. (2008) "Crisi finanziaria, moderno flagello": Aggiornamenti Sociali 59, 671-682.

- Foglizzo, P. (2010) "G20 (Gruppo dei 20)": Aggiornamenti Sociali 61 , 463-466.

- PalaIS-ROYAl INITIATIVE (2011) Reform of the international monetary system: a cooperative approach for the twenty first century (8 de febrero), http://global-currencies.org/smi/gb/telechar/news/ Rapport Camdessus-integral.pdf

- Pontificio consejo justiCIA y PAz (1994a) Aspetti social ed etici dell'economia. Un colloquio in Vaticano (Actas del primer Seminario de economistas, organizado el 5 de noviembre de 1990), Ciudad del Vaticano, Libreria Vaticana.

- PontiFicio consejo Justicia y paz (1994b) World Development and Economic Institutionss (Actas del segundo Seminario de economistas, organizado el 4 de enero de 1993), Ciudad del Vaticano, Libreria Vaticana.

- de Salins A. y de Villeroy de Galhau, F. (1994) II moderno sviluppo delle attività finanziarie alla luce delle esigenze etiche del cristianesimo, Ciudad del Vaticano, Libreria Vaticana.

- Salvini, G. (2011) "Una riflessione sulla riforma del sistema finanziario e monetario": La Civiltó cattolica 162-IV, 487-493.

- SibleY, A. (2001) Financial Reform: the errors of the Vatican's critics (4 de noviembre). www.speroforum.com/aJYMKMPQTLC56/Financial-Reform-the-errors-of-the-Vaticans-critics.

- Toso, M. (2012) Reflexiones sobre la reforma del sistema monetario y financiero (3 de enero). http://pcgp.it/dati/2011-12/28-999999/NRSFLREFLEXIONES.pdf

- Oficina nacional de la Conferencia Episcopal Italiana para los problemas sociales y del trabajo (2000) Etica e finanza.

- Oficina nacional de la Conferencia Episcopal Italiana para los problemas sociales y del trabajo (2004) Finanza internazionale e agire morale.

- Oficina nacional de la Conferencia Episcopal Italiana para los problemas sociales y del trabajo (2006) Etica, sviluppo e finanza. www.chiesacattolica.it/cci_new/documenti_cei/2006-11/14-36/ EticaSviluppoFinanza.pdf 